\title{
Le laboratoire de dosimétrie passive de l'établissement COGEMA/AREVA de La Hague
}

\author{
H. TRUFFERT
}

\subsection{Le dosimètre photographique, le temps des films...}

L'histoire de la dosimétrie de l'établissement de La Hague pour le personnel travaillant sur le site (CEA puis COGEMA puis AREVA et les Entreprises sous-traitantes) commence à la fin de l'année 1965, cela correspond aux débuts de l'usine. Autant dire que ce sont les balbutiements pour la technique et pour les hommes qui ne sont pas encore des spécialistes du domaine. Comme partout en France, le dosimètre utilisé alors est le film, avec la technique de développement et de lecture identiques à ce qui se fait partout dans les autres laboratoires, en particulier au Service technique d'étude et de protection (STEP) du CEA de Fontenay-aux-Roses qui assurait la dosimétrie des personnels affectés à La Hague avant 1966.

Le dosimètre photographique de l'époque est une émulsion Dupont de Nemours et ce jusqu'en 1970 (figure 11.1). Cette émulsion sera remplacée par un film Kodak type 3, lui-même remplacé par le Kodak type 2 à partir de 1998 jusqu'à la fin de l'utilisation de cette technique dosimétrique. Le dosimètre photographique est tenu dans une pince fournissant une mesure sous une plage « nue », sous un écran d'étain et sous un écran de cadmium. Les deux premières plages permettent d'évaluer les composantes $\beta$ et $\gamma$, l'écran de cadmium étant destiné, en théorie, à la mesure des neutrons. Ce premier « badge » sera utilisé jusqu'en 1968.

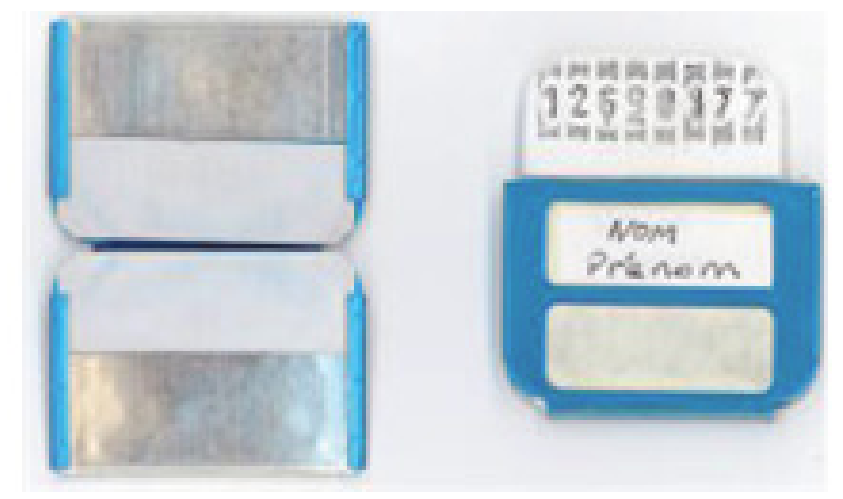

Figure 11.1 - Premier dosimètre photographique COGEMA. 
À partir de cette date, les films Dupont de Nemours puis le Kodak type 3, seront placés dans un boitier «DM6» (dosimètre Marcoule « 6 plages ») (figure 11.2). L'objectif de ce jeu d'écrans de natures différentes est d'appréhender la nature des rayonnements incidents et de définir ainsi le mode de calcul des différents équivalents de doses. Nous trouvons dans ce boitier une plage «nue » (pas d'écran pour les bétas), un écran de polyéthylène, d'aluminium, de cadmium, d'étain, d'étain + plomb, tous de masse surfacique différente. Ce boitier associé au film Kodak type 3 sera en service à La Hague jusqu'en 1986.

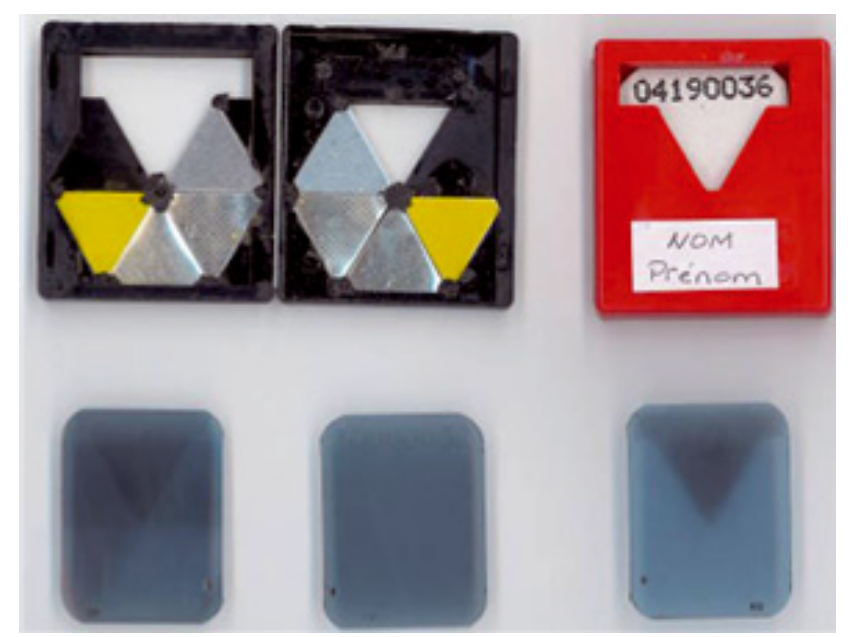

Figure 11.2 - Dosimètre photographique dans le boitier « DM6 ».

L'exploitation du boitier « DM6 » et du film est totalement manuelle, à commencer par le marquage du film qui se fait film par film au moyen d'un composteur automatique («l'Automator ») dont le réglage est des plus délicats (figure 11.3).

La numérotation se fait sur l'enveloppe papier qui protège l'émulsion. Il faut une pression suffisante pour pouvoir lire le numéro après développement du film, mais pas trop forte afin de ne pas percer l'enveloppe ce qui rendrait la lecture du numéro impossible et dans le pire des cas celle de l'émulsion dans sa totalité.

Chaque boitier est ouvert à la main pour y introduire ou retirer le film. Chaque boitier est identifié par les nom et prénom du porteur au moyen d'une étiquette collée à la main et frappée à la machine à écrire, ce qui vaut parfois quelques énervements, bien compréhensibles, de la part de la secrétaire. En effet, très souvent les étiquettes 


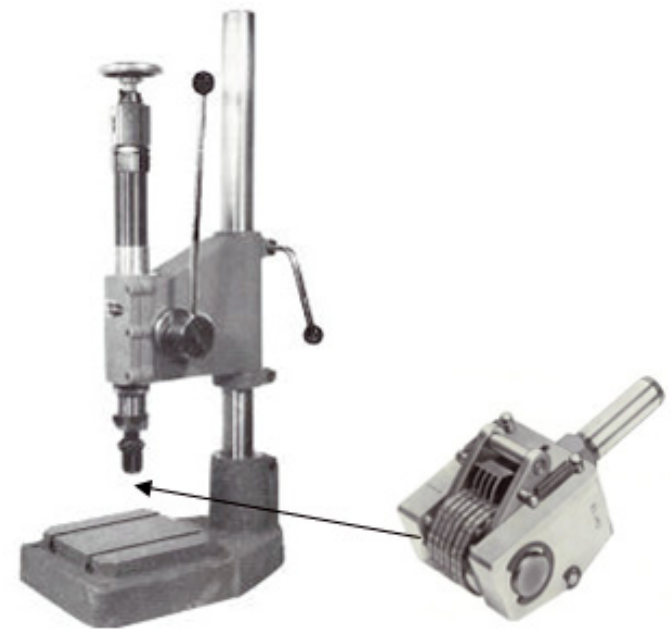

Figure 11.3 - Dispositif de numérotation des dosimètres photographiques par pression.

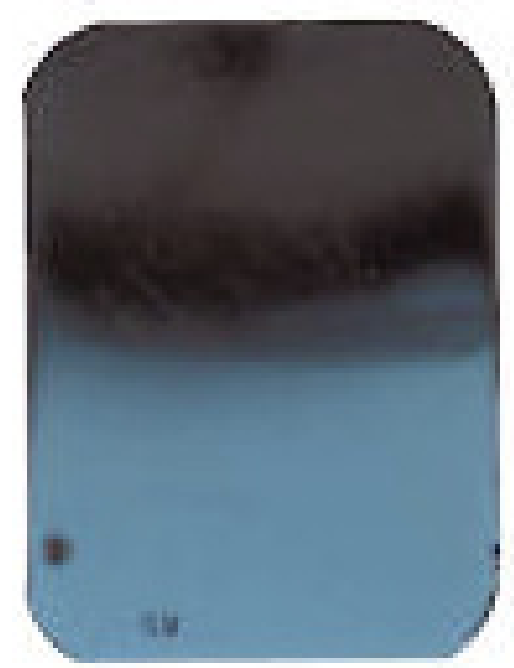

Figure 11.4 - Dosimètre photographique dont l'enveloppe a été percée lors de la numérotation. 
H. TRUFFERT

se décollent de leur support et viennent bloquer le mécanisme de la machine à écrire ! Chaque boitier est glissé dans une enveloppe de vinyle, pochette qui est ensuite soudée au moyen d'une soudeuse du même type que celles qui sont utilisées pour souder les manches vinyles de boites à gants. Quand tous les dosimètres sont préparés, ils sont ensuite triés dans des tableaux nominatifs, à la manière du facteur qui triait ses lettres avant de partir faire sa tournée. Les tableaux, munis des dosimètres témoins sont ensuite disposés dans les différentes installations de l'usine sur les différents lieux de passage des personnels, en général près des vestiaires. Au retour des dosimètres, afin de minimiser les risques d'erreur d'identification, ils sont classés par ordre numérique croissant par lot de 20 dans des boites de classement de 25 alvéoles. Les étuis sont alors découpés au ciseau (c'est encore le cas en 2010 !), les films séparés des boitiers et placés toujours en ordre numérique dans d'autres boites pouvant contenir 400 films. Avant de passer au développement, les films sont attachés avec un élastique par paquets de 10 toujours afin d'assurer le «bon ordre» au cours du développement. Des films «tests» et des films d'étalonnage (émulsions vierges ou irradiées à une dose connue) sont disposés entre les dosimètres des salariés, aux endroits appropriés et à intervalles réguliers. L'objectif de ces points que l'on appellerait aujourd'hui «points qualités » étant de s'assurer de l'homogénéité du développement (de la chimie du développement) et de contrôler les appareils de mesure tout au long de la lecture de l'ensemble des dosimètres.

C'était ensuite l'entrée en chambre noire, de toute l'équipe du laboratoire, y compris le chef du laboratoire ! Seule une lampe inactinique est allumée. Il faut environ 15 minutes avant de commencer à voir les boites, les paquets de films et les paniers de réception des émulsions photographiques.

Les premiers paniers de développement sont verticaux, en inox et peuvent recevoir 200 émulsions. La dernière génération de paniers sera en «PVC ». Ces paniers sont horizontaux, carrés et peuvent recevoir 400 émulsions. Jusqu'en 1980, les paniers verticaux sont utilisés, il faut les plonger un par un, successivement dans le bain de développement, le bain d'arrêt, fixateur et bain mouillant. À l'arrivée des paniers de 400 alvéoles, on plongera 11 paniers soit 4400 émulsions en une seule fois dans chacun des bains. Un palan électrique sera mis en place à l'occasion pour réaliser cette plongée. Pendant le trempage des émulsions, les bains sont agités au moyen d'un flux d'azote afin d'en assurer l'homogénéité. Les différents temps de trempage sont mesurés au moyen d'un chronomètre pouvant être lu sans lumière, mais sans risque de noircir les émulsions non encore développées. Le bain mouillant (eau avec du «produit à vaisselle ») est utilisé pour minimiser les traces sur les émulsions. De plus les émulsions sont rapidement placées dans une armoire séchante, là aussi afin d'obtenir des émulsions vierges de toute trace de séchage. 
À l'époque du film Kodak type 3 il y a deux émulsions dans l'enveloppe papier, une émulsion sensible dite « rapide» et une moins sensible dite « lente». Seule la première émulsion est développée, la deuxième est replacée dans l'enveloppe papier, les paquets de 10 reconstitués (au moyen de l'élastique) et remis dans la boite. Les boites contenant les «deuxièmes émulsions » seront placées dans un meuble étanche à la lumière. On ne retournera en chambre noire développer une $2^{\mathrm{e}}$ émulsion que si la $1^{\text {re }}$ est saturée (forte dose) ou pose un problème de lecture. Autant dire que la $2^{\mathrm{e}}$ émulsion sera très rarement utilisée. Cette façon de faire n' aura plus lieu d'être avec l'utilisation du Kodak type 2 puisque les émulsions sont sur le même support et que c'est par grattage dans l'eau tiède que l'on atteint l'émulsion la moins sensible. Il faut environ trois heures pour traiter 4500 films. Le temps passé en chambre noire est un moment convivial, même si parfois un cri de douleur fuse dans la chambre noire : une enveloppe papier récalcitrante à l'ouverture fait qu'une personne de l'équipe s'est glissé une émulsion sous un ongle. Il était préférable d'avoir les ongles un peu longs le jour du développement! Au jeu de celui qui finirait son panier de 400 films le plus rapidement, souvent le personnel féminin sortait vainqueur! Après le séchage, les films sont placés sur des dépliants de 150 films afin d'être lus. La lecture est effectuée au moyen d'un densitomètre à transmission, en l'occurrence un « Digitalog » (figure 11.5).

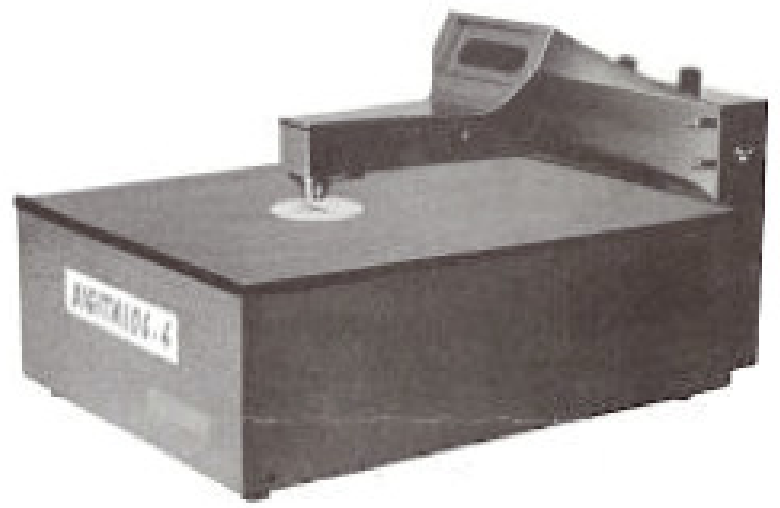

Figure 11.5 - Densitomètre à transmission «Digitalog » de la marque Garreau.

L'intensité lumineuse transmise détermine la densité optique (le noircissement de l'émulsion) directement liée à la dose reçue par le film. L'opérateur au poste de lecture a devant lui son dépliant de 150 films. Dans un premier temps, il vérifie la numérotation, s'assure que la suite est logique (incrémentation de un), sinon les 
numéros manquants sont renseignés comme dosimètres non rendus. L'opérateur extrait du dépliant les dosimètres qui présentent un voile gris plus foncé que l'émulsion vierge. Ce poste nécessite donc une certaine compétence. Les trois plages (dosimètre à pince du début) ou les six plages pour le boitier DM6 sont lues une à une sous le densitomètre.

Plus tard, un densitomètre plus sophistiqué permettra de lire les six plages en un seul positionnement de l'émulsion. Le passage de la densité optique à la dose apparente se fait au travers d'une courbe d'étalonnage établie à partir d'émulsions issues du même lot que celui des films portés par le personnel et irradiés à des doses connues de 15 mrem $(0,15 \mathrm{mSv})$ à $4 \mathrm{rem}(40 \mathrm{mSv})$. Les sources d'étalonnage utilisées sont, selon les époques, du radium 226, du cobalt 60 ou du césium 137.

Au début (années 1970 à 1980), à côté de la personne au poste de lecture se trouve un autre opérateur. Il note à la main les valeurs des densités optiques que lui dicte son collègue sur une fiche spécifique où sont prérenseignés le nom, prénom, lieu de travail du salarié ainsi que l'intitulé des différentes plages du boitier (Poly, alu, Sn, Sn Pb, nue, Cd). Ces fiches sont ensuite envoyées au service de «mécanographie » où sont créées des fiches perforées à partir desquelles seront effectués les calculs de dose et l'attribution de la dose au salarié. L'ère de l'informatique n'existait pas encore! Outre le passage des émulsions sous le densitomètre, l'opérateur a également la mission de déterminer le code de la formule qui doit être utilisée pour le calcul de la dose. Le code 0 pour les irradiations $\beta$, les codes 1,3 , pour les irradiations $X$ (plutonium principalement), le code 2 pour une irradiation $g$ de forte énergie, le code 4 pour une irradiation $\gamma$ de faible énergie, le code 5 pour un mélange d'irradiation $\beta, \gamma \ldots$ pour les principales. Les calculs issus de ces formules permettent ensuite de définir l'équivalent de dose « au corps entier », à la peau ou au cristallin. Ce sont les images vues sur le film (noircissement selon l'atténuation des différents écrans du boitier en fonction de l'énergie et la nature du rayonnement) qui permettent à l'opérateur de déterminer la nature de l'irradiation reçue par le porteur (figure 11.6).

L'opérateur note aussi toute anomalie constatée sur l'émulsion. Ces anomalies dites «d'irradiation» sont signalées par une lettre par exemple ( $\mathrm{H}$ : film collé, lecture impossible, $\mathrm{L}$ : traces d'écran anormales, $\mathrm{M}$ : tache de contamination, $\mathrm{O}$ : film irradié à l'envers, $\mathrm{R}$ : anomalie, consulter les archives...). Cette opération de lecture de films demande une certaine compétence de l'opérateur !

Après 1980, l'opérateur se retrouvera seul face au densitomètre et aux films. Les données sont alors enregistrées directement sur disquettes (il y en aura de différentes tailles...) pour finir par une liaison directe entre les PC reliés au densitomètre et les serveurs informatiques qui traitent les données. 


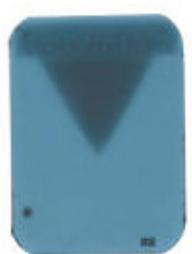

Irradiations :

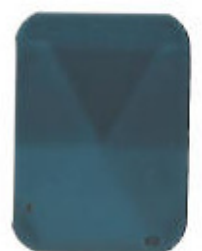

$\mathrm{X}$ de faible énergie

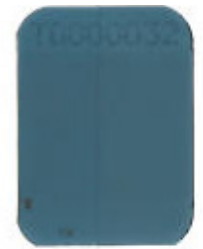

y de forte énergie

Figure 11.6 - Images correspondant à des expositions à des $\beta, X$ et $\gamma$.

Le principal avantage du film reste « l'information visuelle » qu'il donne après développement. En effet l'œil « expert », en fonction des différents noircissements laissés par les écrans du boitier (ou l'uniformité du noircissement) peut, dans la majorité des cas, connaître la nature du rayonnement. Information importante pour le calcul des doses, la confirmation de la dose ou l'interprétation des événements qu'aurait subit le dosimètre (exemple : l'image d'une lame de scie à métaux sur le film tend à prouver que le dosimètre n'était pas sur le porteur au moment de l'irradiation mais plutôt dans la caisse à outils, le passage du dosimètre en machine à laver le linge !)

L'hypersensibilité de l'émulsion photographique s'avère être le principal inconvénient du film. En effet dans les basses énergies $X$ ou $\gamma$ (plutonium, américium par exemple) le film peut présenter une réponse en énergie 30 fois trop élevée, ce qui oblige à faire des corrections très importantes sur la valeur des densités optiques et nuit à la fiabilité de la mesure de la dose (figure 11.7).

En complément de cette dosimétrie film permettant d'effectuer la mesure des rayonnements photoniques et bêtas il faut, sur une usine telle que celle de La Hague, réaliser également une dosimétrie des neutrons. C'est l'utilisation obligée d'un autre type de détecteur.

\subsection{La dosimétrie des neutrons, le début de la thermoluminescence...}

De 1968 à 1976 la mesure des neutrons est réalisée à l'aide des émulsions Kodak type A (NTA). Ces plaques sont introduites dans une pochette aluminium qui après avoir été soudée est placée derrière le boitier contenant le film. 


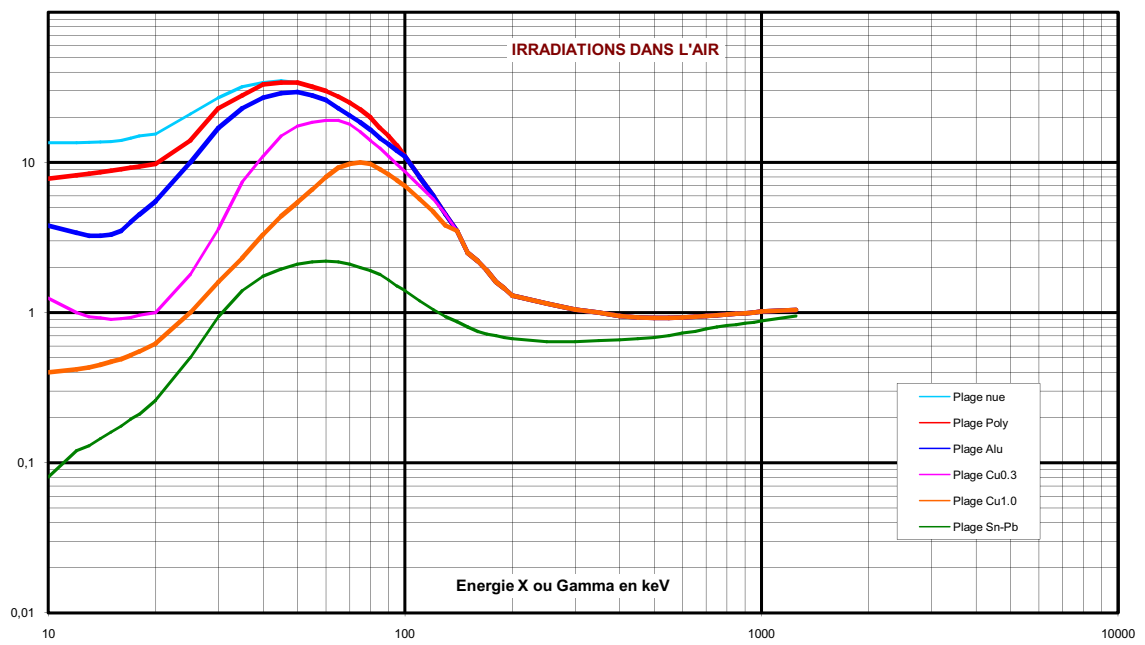

Figure 11.7 - Réponse relative du film en fonction de l'énergie des X ou $\gamma$ sous différents écrans.

La performance de ce dosimètre est peu adaptée au regard des seuils à détecter et des spectres à évaluer. De plus la technique de lecture est archaïque. Après le développement de l'émulsion, chaque plaque nucléaire est lue sous un microscope. Les traces (trous dans l'émulsion) laissées par les protons sont comptées. La dose est en théorie proportionnelle à ce nombre de traces. Autant dire que le bilan dosimétrique est quasiment nul... Certains avouent attraper le « mal de mer » à lire les plaques nucléaires, les yeux rivés sur les oculaires du microscope alors que l'on déplace les émulsions sur la platine pour chercher les traces.

Depuis 1976, la dosimétrie des neutrons est déduite de l'exploitation de cartes munies de dosimètres thermoluminescents. Jusqu'à fin 1989 seuls les salariés affectés sur des ateliers bien ciblés sont équipés. Ce sont les ateliers de réception des combustibles et de traitement du plutonium. Les unités d'intervention (décontamination, mécanique) sont également équipées. Depuis 1990 tous les salariés du site sont équipés de cartes thermoluminescentes. 


\subsection{Le développement du laboratoire de dosimétrie}

\subsubsection{Le développement du site, des usines spécifiques à surveiller...}

La vocation de l'usine AREVA NC à La Hague est de traiter pour les recycler les combustibles nucléaires usés qui sortent des réacteurs de production d'électricité. C'est la spécificité de ce site qui a conduit à mettre en place un suivi dosimétrique complet et qui explique la politique menée pour le Laboratoire de dosimétrie. La Hague alors «UP2-400» (400 tonnes retraitées par an) accroît sa capacité de retraitement avec le démarrage d'UP3 (1990) puis d'UP2-800 (1994). Il faudra, au début des années 1980, anticiper ces extensions synonymes d'augmentation du personnel destiné à faire fonctionner ces usines et bien sûr qu'il faudra surveiller en dosimétrie externe. Pour ce faire, le laboratoire de dosimétrie va conduire une politique de développements internes que ce soit pour ses moyens d'exploitation ou le dosimètre par exemple. Nous en reparlerons plus loin...

En 1968 le laboratoire de dosimétrie fonctionne avec quatre personnes qui exploitent environ 1500 dosimètres par mois. En 1986, 4500 dosimètres sont traités tous les mois, le laboratoire fonctionne alors avec 12 personnes. Au-delà de l'augmentation du nombre de salariés suivis en dosimétrie externe, l'organisation mise en place anticipe les extensions du site mais également la diversification des missions du laboratoire ainsi que les évolutions techniques.

Le laboratoire a été avant tout conçu pour assurer le suivi dosimétrique des personnes travaillant sur le site, CEA, COGEMA, AREVA ainsi que les personnels des entreprises intervenant sur l'établissement. La spécificité de cette usine au niveau de la radioprotection et en particulier en terme d'irradiation externe réside dans le fait que les champs de rayonnements rencontrés sont multiples et mixtes : $\mathrm{X}, \gamma\left(\mathrm{ex}:{ }^{239} \mathrm{Pu},{ }^{60} \mathrm{Co} \ldots\right.$ ), bétas (ex : ${ }^{85} \mathrm{Kr},{ }^{90} \mathrm{Sr}-\mathrm{Y} \ldots$ ), et neutrons (ex : ateliers plutonium, combustibles usés, déchets vitrifiés), auxquels s'ajoute dans certains ateliers un risque de criticité. Face à cette diversité de rayonnements et la difficulté à obtenir des mesures fiables (sensibilité, seuil de détection...), il fallait faire évoluer les systèmes et donc évaluer d'autres techniques. Pour s'en donner les moyens, une métrologie des rayonnements associée à la dosimétrie devint nécessaire.

\subsubsection{La participation aux GT des laboratoires français, la métrologie des rayonnements au laboratoire}

La nécessité d'évoluer et de mettre en place une métrologie des rayonnements conditionnera l'organisation future de ce laboratoire et la définition de ses missions. 
H. TRUFFERT

Le laboratoire sera dimensionné pour assurer la dosimétrie des personnels, mission de base, et mener de concert les évolutions.

Les tests de nouveaux dosimètres (pour l'époque) et nouveaux matériels débutera à la fin des années 1970 pour se poursuivre jusqu'en 2000. Pour ce faire, une fonction « métrologie des rayonnements » sera créée au sein de l'équipe, cette organisation est toujours d'actualité en 2010 ... Il n'y aura pas que la technique qui motivera les changements mais aussi les recommandations internationales (ICRP, ICRU) et la règlementation (directives européennes, arrêtés, décrets, code du travail...).

Cette progression, le laboratoire ne va pas la faire seul. C'est en participant aux travaux des Groupes de travail français que le laboratoire va évoluer. À ces GT (GT 5, GTN1, GT Criticité...) participent les laboratoires des SPR français (CEA, SDOS, COGEMA, ETCA...), le LMRI et l'IPSN. La principale mission de ces GT consiste à définir une politique commune au niveau français pour l'application des normes et recommandations internationales, pour la normalisation des installations et méthodes d'étalonnage, les expérimentations et la métrologie des rayonnements.

Je citerai pêle-mêle quelques actions communes :

- la conception des irradiateurs photons, bétas et neutrons ;

- la nature des chaines de mesures de références pour l'étalonnage des sources (chambre d'ionisation téflon carbone pour les photons, chambre à cavité variable pour les bétas, la technique multisphères pour les neutrons) ;

- la mise au point des calculs de dose en cas d'accident de criticité, le traitement du SNAC et des verres radiophotoluminescents ;

- les recommandations pour la normalisation des essais physiques des détecteurs et dosimètres bétas ;

- et plus récemment (1999) l'approche des «nouvelles» grandeurs dosimétriques.

Les GT organisent également les intercomparaisons entre laboratoires. Le Laboratoire de La Hague participe activement à ces GT et s'appuie sur les conclusions des différents travaux pour les mettre en applications en termes de métrologie et de dosimétrie individuelle.

Dès la fin des années 1970, la volonté est affichée de doter le laboratoire d'une métrologie des rayonnements parallèlement aux missions d'exploitation des dosimètres individuels. Pour la métrologie des photons le laboratoire s'équipe en 1980 d'un irradiateur type IME 5A chargé de sources de ${ }^{60} \mathrm{Co}$. Ce moyen d'irradiation est essentiellement utilisé pour réaliser les étalons de travail (films, cartes TLD) et tester les différents dosimètres qui sont étudiés. Les références 
d'irradiation des différentes sources de ${ }^{60} \mathrm{Co}$ sont déterminées au moyen de chambres d'ionisation «téflon carbone » associées à un électromètre, ensemble calibré auprès du LMRI. Cette irradiateur sera remplacé par un irradiateur de type Büchler OB20 chargé de sources de ${ }^{137} \mathrm{Cs}$. Le laboratoire utilise cet irradiateur encore aujourd'hui pour ses besoins en étalons de travail et de suivi des cartes TLD utilisées pour réaliser la dosimétrie individuelle et également à des fins d'étalonnages de matériel de radioprotection et de dosimètres opérationnels électroniques.

Pour les études en dosimétrie béta, notamment la réponse aux énergies bétas des différents dosimètres, dès le début des années 1980, le laboratoire s'équipe d'une chambre à extrapolation (chambre à cavité variable) afin d'étalonner les sources de référence qui serviront à qualifier les dosimètres $\left({ }^{147} \mathrm{Pm},{ }^{204} \mathrm{Tl},{ }^{90} \mathrm{Sr} / \mathrm{Y}\right.$, $\left.{ }^{106} \mathrm{Ru} / \mathrm{Rh}\right)$.

Pour la dosimétrie des neutrons le laboratoire s'équipe en 1980 d'un irradiateur de type IN01 chargé d'une source de ${ }^{241} \mathrm{AmBe}$. Ce système est principalement utilisé pour étalonner les matériels portables de radioprotection et contrôler la présence des TLD au ${ }^{6} \mathrm{LiF}$ sur les cartes TLD. Le calcul de l'équivalent de dose neutrons évoluant avec l'énergie des neutrons, c'est dans les installations nucléaires que le laboratoire effectue ses étalonnages en neutrons. Dès 1976, la technique «multisphères» de Bonner (sphères modératrices en polyéthylène) est mise en œuvre autour des emballages de transports des combustibles irradiés. Les compteurs utilisés pour réaliser la mesure sont des compteurs à l'iodure de lithium ou à l'hélium. La mesure est fastidieuse. C'est avant tout sportif, la sphère de 15 pouces pèse plus de $10 \mathrm{~kg}$, quand les mesures se font en hauteur, deux personnes ne sont pas de trop pour positionner la sphère sur le compteur. Les campagnes de mesures sont rares car les temps de comptages sont longs et le poste de travail ne doit pas évoluer afin que les quatre ou cinq sphères qui sont utilisées mesurent le même spectre. La mesure sur un poste peut durer la journée d'autant plus que les débits d'irradiation sont généralement faibles. Autant dire que l'exploitant de l'installation ne voit pas arriver l'équipe du laboratoire d'un bon œil ! Les calculs des fluences et équivalents de doses se font quasiment «à la main», seuls les laboratoires spécialisés du CEA (Cadarache) ont, à l'époque, les moyens « informatiques » appropriés. La collaboration entre les laboratoires fonctionne bien. En 1995, après une étroite collaboration avec le laboratoire ETCA de l'armée, le laboratoire se dote d'un spectromètre rotatif neutron, le ROSPEC. Six compteurs proportionnels sphériques de différents diamètres (deux ou six pouces) remplis de gaz différents (hydrogène, argon-méthane, hélium) à des pressions différentes sont montés sur une plateforme rotative. Cela permet la mesure en une seule fois et en trois heures tout au plus. Cela nous permettra d'être plus efficace et d'effectuer des mesures sur les différents types de postes de travail où l'irradiation neutron est 
H. TRUFFERT

potentielle. La mesure avec le ROSPEC permet de déterminer le spectre des neutrons en un lieu (comptage par bande d'énergie) et ainsi calculer la fluence neutronique et les équivalents de doses neutrons. Cette mesure dite de « référence » permet d'évaluer le coefficient d'étalonnage des dosimètres passifs qui sont placés au même endroit et dans les mêmes conditions d'irradiation. Cette démarche est appliquée sur les ateliers de réception des combustibles, de traitement du plutonium, de stockage des produits vitrifiés ou encore de confection des combustibles MOX.

La métrologie des rayonnements au laboratoire, que ce soient les photons, les bétas ou les neutrons, si elle sert aussi à des fins de radioprotection aujourd'hui, a été organisée avant tout pour mieux appréhender «la mesure de la dose » : la dosimétrie !

\subsection{Le chemin vers le laboratoire de dosimétrie de 2010}

\subsubsection{Le développement des dosimètres et des machines «La Hague »}

Ces moyens de métrologie des rayonnements seront donc utilisés pour réaliser les essais nécessaires au choix du système dosimétrique et réaliser des mesures aux postes de travail, dans les installations. L'effectif du laboratoire est de 4 personnes en 1975, 10 à partir de 1983. Il sera de 12 personnes en 1994, 13 en 2010. Cette augmentation importante et rapide entre 1975 et 1983 a été motivée par une activité d'exploitation de dosimètres croissante et pour préparer les techniques et les outils nécessaires à assurer le suivi dosimétrique des futurs salariés qui travailleront sur les nouvelles usines UP3 et UP2-800 (10 000 dosimètres par mois seront traités en 1994). De 1980 à 1986, une équipe du laboratoire va, parallèlement à l'exploitation des dosimètres, réaliser différentes études en dosimétrie et concevoir les matériels d'exploitation qui façonneront la structure du laboratoire telle qu'elle est encore aujourd'hui.

Trois axes de développement sont nécessaires pour répondre à la fois à la croissance du site et à la spécificité des risques radiologiques. Il faut améliorer les performances en dosimétrie des neutrons et des bétas, « automatiser » l'exploitation des dosimètres et développer une dosimétrie «poignet» autre qu'un boitier « poitrine » avec un bracelet (peu pratique pour les personnes qui ont à le porter).

\subsubsection{Le choix du système de dosimétrie poitrine}

Pour réaliser la dosimétrie des neutrons et pallier les faiblesses des émulsions photographiques, dans les années 1980, il n'y a pas d'autre choix que les dosimètres thermoluminescents (TLD). Le laboratoire va étudier plusieurs types de TLD. Le 
cahier des charges est simple. Il faut pouvoir mesurer les neutrons, donc le fluorure de lithium-6 s'impose.

La mesure des bétas est un critère indispensable également. Le laboratoire réalisera à cette époque une étude approfondie de la réponse de plusieurs TLD aux rayonnements bétas. Enfin il faut que les dosimètres puissent être produits en série sans que les caractéristiques changent. Au début des années 1980, les industriels capables d'une telle performance ne sont pas nombreux !

Dès 1975, le laboratoire travaillera sur les dosimètres thermoluminescents. Ce sont d'abord des dosimètres composés d'un mélange de téflon et de fluorure de lithium que l'on découpe à la main et que l'on identifie au crayon à papier. Cette période fut surtout propice pour apprendre la technique de la thermoluminescence : définition des programmes de chauffage des dosimètres, maitrise du lecteur (tous les réglages se faisaient à la main au moyen de potentiomètres). Puis apparurent sur le marché les dosimètres thermoluminescents «PTL717 » (fluorure de lithium enrichi à 99,95\% ${ }^{7} \mathrm{Li}$ ) mis au point par Gui Portal et ses équipes. On progressait lentement au niveau de la reproductibilité des différents lots produits. Le numéro atomique de ce matériau proche de celui des «tissus mous » permet d'obtenir de faibles variations avec l'énergie du rayonnement. Ces dosimètres seront principalement utilisés pour évaluer des débits de doses dans des endroits où l'homme n'a pas accès. Ils seront mis également aux doigts lors de certaines interventions. Les dosimètres sont identifiés individuellement à l'encre («Rotring ») et chauffés individuellement. Le lecteur est encore manuel, mais les réglages plus accessibles (figure 11.8).

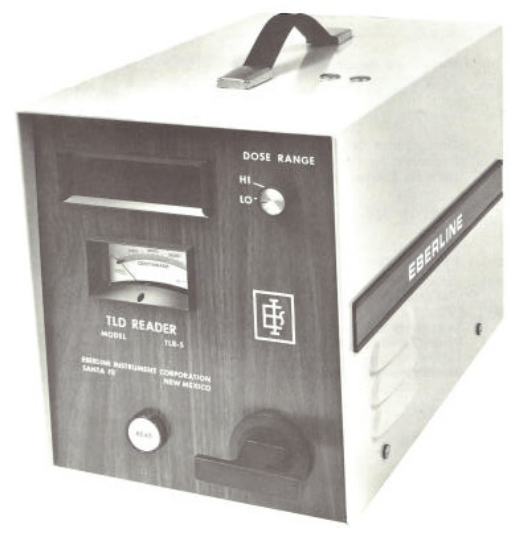

Figure 11.8 - Lecteur de dosimètres thermoluminescents. 
Le ${ }^{7} \mathrm{LiF}$ seul ne permet pas de réaliser la dosimétrie des neutrons. Il faut lui associer un dosimètre de Li6 et créer un dosimètre à albédo basé sur la différence ${ }^{6} \mathrm{LiF},{ }^{7} \mathrm{LiF}$.

Après les émulsions Kodak NTA, en 1976 la dosimétrie sera réalisée au laboratoire avec des TLD 700 et TLD 600 fabriqués aux États-Unis par la société HARSHAW. Les deux dosimètres sont placés sur une carte métallique de fabrication Harshaw (appelée « carte thermoluminescente » ou « carte TLD » dans le langage courant). L'identification se fait par un « code à trous ». Le remplacement des plaques nucléaires par les cartes thermoluminescentes va se faire progressivement. La carte Harshaw évoluera de deux à quatre dosimètres, 2 TLD 600 et 2 TLD 700. L'identification de la carte se fera alors au moyen d'un code à barres. Jusqu'en 1989, les personnels travaillant dans les ateliers de réception des combustibles, ceux des ateliers du traitement du plutonium et les équipes d'intervention (mécanique, assainissement) seront équipés. En 1990, tout le personnel suivi par le laboratoire sera équipé de cartes TLD. Ces cartes sont encore placées dans une pochette en aluminium, soudée et positionnée derrière le boitier DM6 jusqu'en 1986 et directement dans le badge «LH2 » ensuite. De 1976 à 1990, la population surveillée en dosimétrie neutrons augmente (de 250 à 7000 personnes par mois). Les cartes TLD évolueront au fil du temps. Toujours un couple TLD 600 et 700 mais le concept de la carte Harshaw rivetée change. Sur cette carte tous les cristaux sont solidaires, si l'un des TLD est défectueux, c'est la carte entière qui est réformée. Le concept français de cristaux conditionnés sous forme de clips permet de n'écarter que le TLD hors service. Quand les objets se comptent par plusieurs dizaines de milliers l'économie n'est pas négligeable ! Ce concept sera repris pour le dosimètre poignet $« \operatorname{extREM}^{\circledR}{ }^{\circledR}$.

Le dosimètre n'est pas le seul élément à prendre en compte pour équiper le laboratoire. Il faut choisir le lecteur automatique de dosimètres. Trois systèmes (TLD + lecteur) seront étudiés, celui des sociétés Alnor, Vinten et Harshaw. Le laboratoire s'équipera en 1979 d'un lecteur semi-automatique (autonomie de lecture de 150 cartes TLD) de type Harshaw 2276, puis deux lecteurs de type Vinten 814 présentant une cadence plus élevée et d'une technologie plus avancée. Ces lecteurs de dosimètres ont été utilisés jusque 1986, date à laquelle le lecteur Harshaw $8000 \mathrm{C}$ (chauffage par doigt de chauffage au contact du dosimètre) sera mis en service. Les quatre dosimètres sont chauffés simultanément, l'autonomie est de 1400 cartes. Le choix se fera sur un compromis entre les performances techniques du lecteur, des dosimètres et le coût. Les TLD Harshaw (cartes TLD composées de $2^{7} \mathrm{LiF}$ et ${ }^{6} \mathrm{LiF}$ ) et le lecteur Harshaw seront choisis (figure 11.9).

Le lecteur Harshaw 8000 C sera remplacé par un lecteur Harshaw 8800 en 1988. La principale différence provient du système de chauffage plus performant. Les 


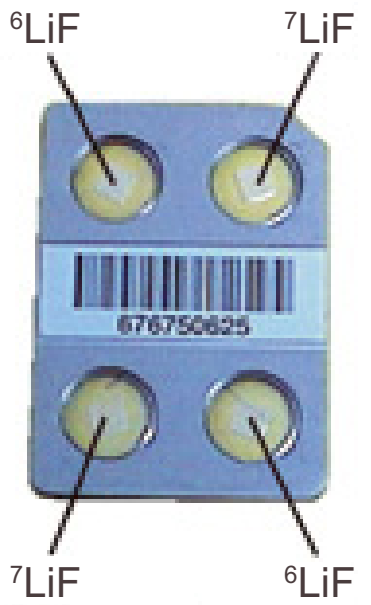

Figure 11.9 - Carte TLD Harshaw avec quatre pastilles thermoluminescentes.

dosimètres sont chauffés au moyen d'azote (gaz), suppression du contact mécanique sur le téflon qui enrobe les dosimètres, pas de déformation, ce qui augment la durée de vie des cartes TLD. Dès 1997, 4 lecteurs Harshaw 8800 équipent le laboratoire (figure 11.10).

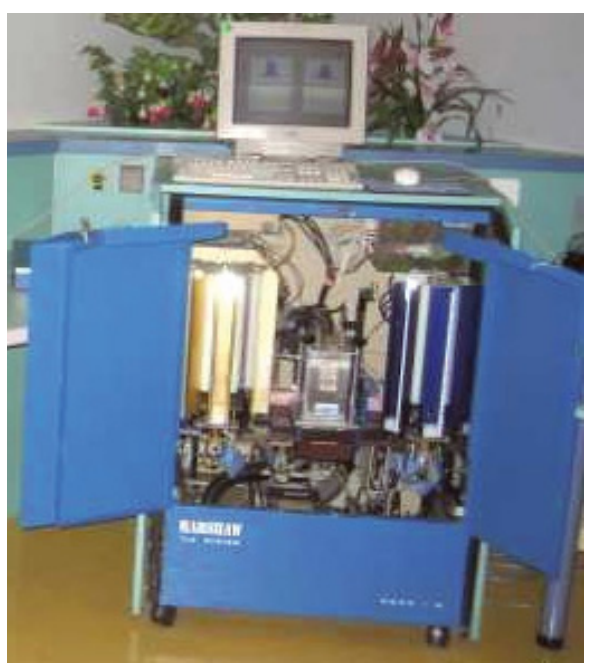

Figure 11.10 - Lecteur de dosimètres thermolumiescents Harshaw 8800. 
H. TRUFFERT

\subsubsection{Le choix du système de dosimétrie poignet}

De nombreux métiers exercés sur le site nécessitent le port d'un dosimètre au poignet en complément du dosimètre poitrine et des dosimètres fixés en bout de doigt. Ce sont principalement les personnes qui travaillent en boites à gants, les personnes qui effectuent la maintenance et la décontamination. Jusqu'en 1984, le dosimètre poignet est constitué d'un boitier DM6 et d'un film conditionné dans un étui comportant deux boutonnières afin de pouvoir utiliser un bracelet. L'objet est volumineux, il bride le porteur dans ses mouvements (entre autre lié au port de gants de protection) ce qui fait qu'il est peu porté. Dans ces conditions, l'objectif d'un suivi de la dose au poignet n'est pas atteint !

Le laboratoire étudiera en 1984 un nouveau dosimètre poignet de petite taille avec de meilleures performances techniques. C'est un dosimètre muni de 2 TLD identiques à la carte TLD poitrine $\left(1{ }^{6} \mathrm{LiF}\right.$ et $\left.{ }^{7} \mathrm{LiF}\right)$. Cette « minicarte » sera placée dans un badge comprenant un écran $\mathrm{ABS}$ et une plage «nue ».Pour lire les dosimètres la « minicarte » sera placée sur une carte au format de la carte utilisée avec le dosimètre poitrine afin de pouvoir utiliser le lecteur Harshaw 8800. La fixation de la «minicarte » sur la carte support se fait au moyen d'une semelle aimantée (figure 11.11).
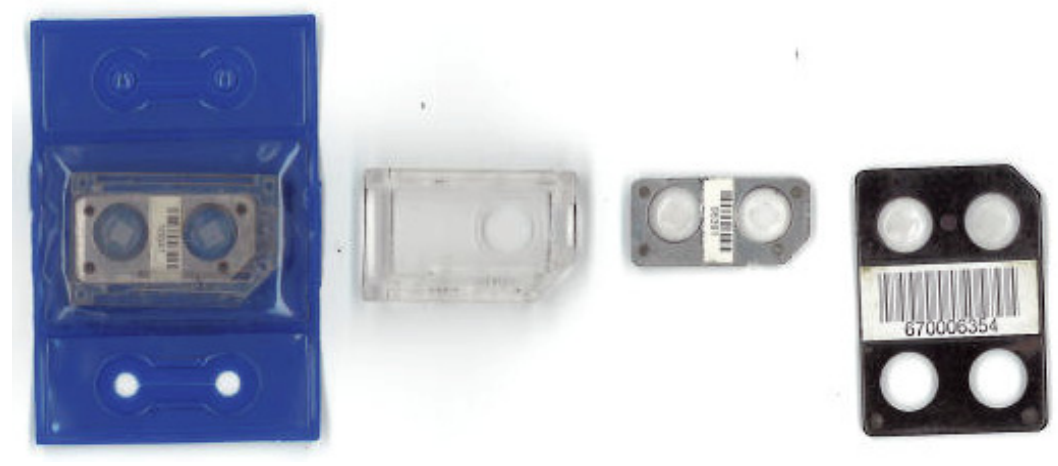

Figure 11.11 - Dosimètre de poignet.

En 1986, le dosimètre poignet évolue (figure 11.12). Si les mêmes dosimètres sont utilisés, le badge et la «minicarte » changent, les principes de fixation également. 


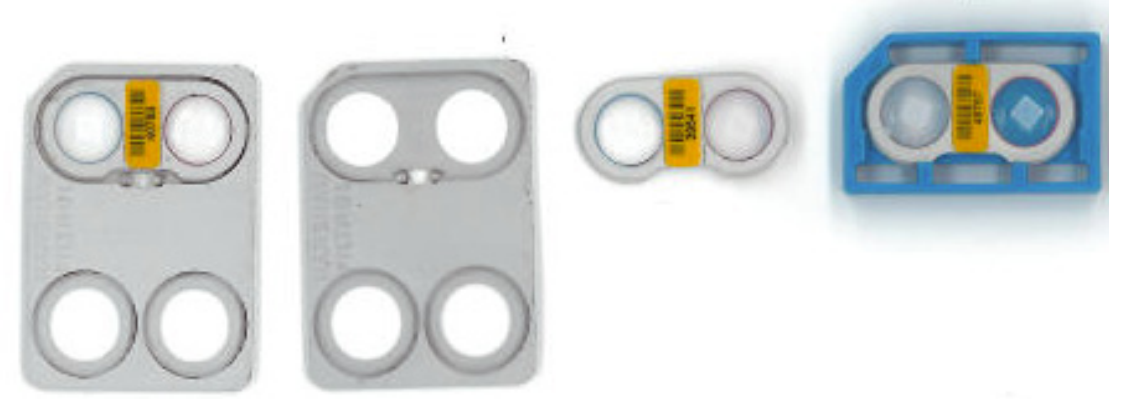

Figure 11.12 - Dosimètre de poignet modèle 1986.

Ces changements sont motivés par le fait que la machine utilisée pour exploiter ces dosimètres (chargement du dosimètre dans le badge, déchargement du badge et positionnement sur la carte support pour lecture) n'a pas le fonctionnement escompté. Le deuxième système et la deuxième machine seront plus performants, ils seront en service jusqu'en 2002. À noter que si les dosimètres (TLD700 et TLD600) proviennent des États-Unis, le laboratoire s'appuie sur une société française, pour la conception de la mécanique entourant les dosimètres, pour les badges et les machines d'exploitation.

Afin de pouvoir absorber l'augmentation de « la production » de dosimètres à effectif constant il faut impérativement automatiser l'exploitation : ne plus ouvrir le boitier à la main pour y mettre ou enlever l'émulsion photographique, ne plus conditionner à la main les plaques nucléaires ou les cartes TLD ou enfin ne plus souder à la main les étuis vinyles qui protègent les dosimètres.

Pour la dosimétrie poignet dès 1984 à la mise en service du dosimètre TLD, la chaîne de fabrication est automatisée avec plus ou moins de réussite car, comme nous l'avons vu plus haut, la première génération de dosimètres poignet et sa machine d'exploitation n'ont pas le rendement attendu. Pour la deuxième génération de dosimètres poignets, la précision demandée au fonctionnement de la machine pour clipser et déclipser la minicarte fait que le système est perfectible. Fort de ces expériences et de la collaboration avec la société travaillant sur le sujet depuis de nombreuses années, la troisième génération de dosimètres poignet sera

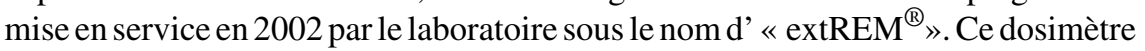
sera conçu pour être exploité manuellement ! Il faudra au laboratoire une bonne dose de persuasion pour faire admettre cet investissement à contre-courant de l'automatisation engagée... Le dosimètre est en une seule partie, boitier et TLD indissociables. En pliant (à la main) le dosimètre se trouve dans les conditions de port, en dépliant, en position de lecture. Seul le conditionnement en pochette PVC 
est automatisé. Ce système est encore utilisé en 2010. D’autres laboratoires ont repris ce système et l'utilisent également en dosimétrie poignet (figure 11.13).
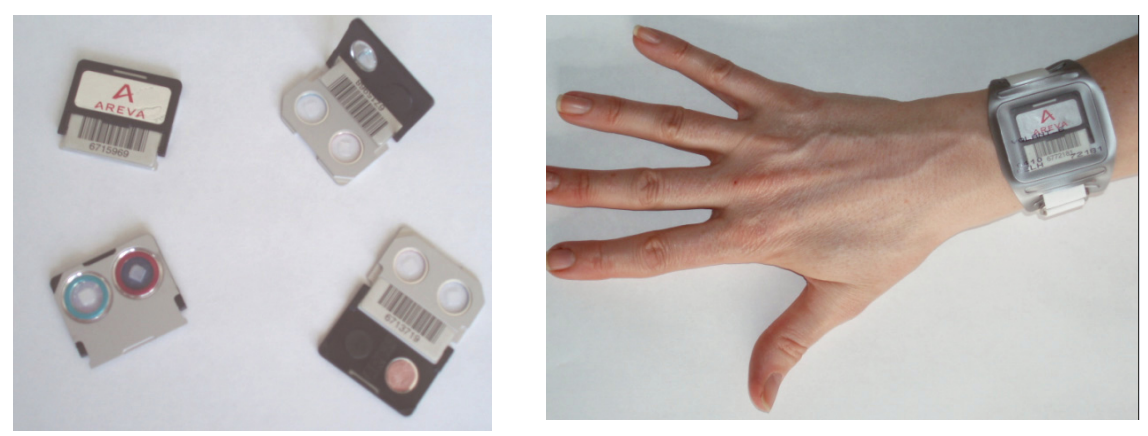

Figure 11.13 - Dosimètre poignet conditionné et porté.

\subsubsection{Le Cogebadge ${ }^{\circledR}$...}

La grande évolution du laboratoire reste toutefois l'abandon du boitier DM6 et son remplacement par un boitier adapté aux besoins de l'établissement et entièrement automatisable (figure 11.14). La préétude de ce badge, identifié «LH2 » dans un premier temps, (badge La Hague $n^{\circ} 2$ ) débute en 1980. Il sera mis en exploitation à partir de 1987. Ce badge est conçu pour recevoir un film et une carte TLD. Les deux dosimètres sont introduits et retirés au moyen d'une machine spécialement conçue à cet effet.

Dans une première phase la mise au point de ce badge a consisté à déterminer la nature et l'épaisseur des écrans à disposer devant le film et devant la carte TLD et incorporer dans ce boitier les dosimètres à activation destinés à l'évaluation des doses en cas d'accident de criticité. Cela en fait un badge complet pour évaluer les doses provenant de rayonnements de toutes natures : une plage sans écran («plage nue ») devant le film et un TLD 700 pour les rayonnements de faibles énergies (bétas), un écran en ABS devant le film et un TLD 600, différents écrans métalliques (cuivre, aluminium, étain, plomb) afin d'identifier les différentes énergies X et gammas et des écrans de cadmium devant les TLD pour la dosimétrie neutrons. Des cibles activables d'ébonite et d'or sont placées à l'arrière du badge pour la dosimétrie de criticité. Ces cibles sont identiques en tout point à celles que l'on trouvait dans le boitier PS2 C (évolution du PS1C) de l'IPSN pour la dosimétrie du CEA afin de pouvoir assurer une entraide en cas d'accident de criticité en France. 


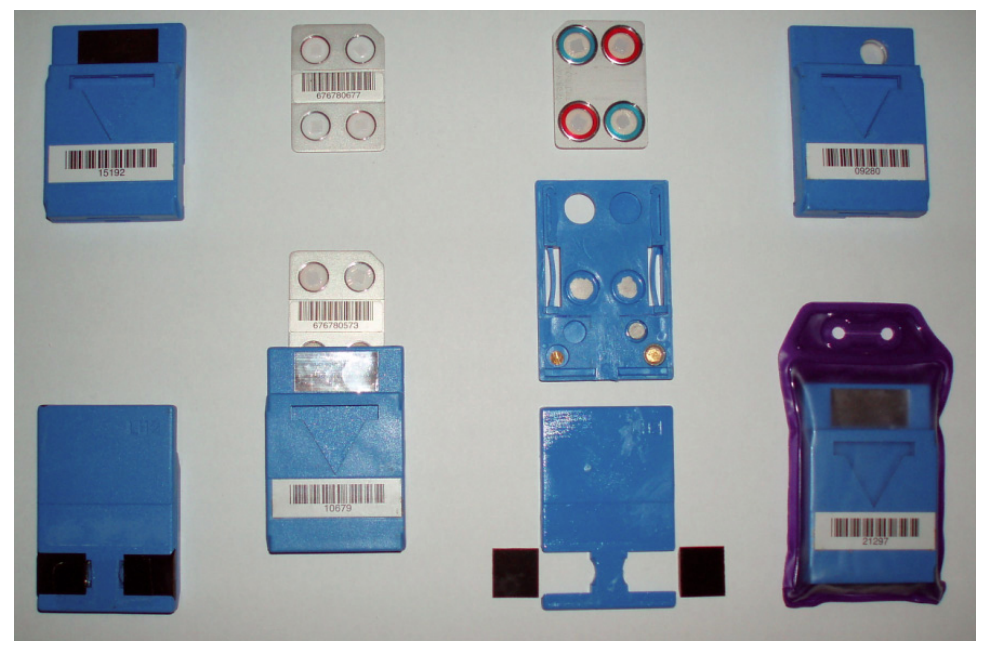

Figure 11.14 - Le dosimètre Cogebadge.

La structure du boitier étant définie, des essais d'irradiation ont été réalisés dans une deuxième phase. Ces irradiations en X et gammas monoénergétiques se sont déroulés en collaboration avec l'IPSN/DPHD/SDOS d'abord en terme de dose dans les tissus sous $300 \mathrm{mg} . \mathrm{cm}^{-2}$ (Dt3), grandeur en vigueur en 1985 puis en terme de $H p(10)$ et Hp (0.07) en 1995 à l'arrivée des recommandations internationales et des « nouvelles » grandeurs opérationnelles.

Au-delà du test du comportement du film et de la carte TLD dans ce boitier, ces qualifications en irradiations ont servis à mettre au point les algorithmes de calculs des différentes grandeurs de référence et autres équivalents de doses. Ces irradiations seront complétées par des études à différents postes de travail dans les ateliers de l'usine. À sa mise en service, ce boitier et les dosimètres qu'il contient sera exploité sous le nom de Cogebadge ${ }^{\circledR}$. Le Cogebadge ${ }^{\circledR}$ ainsi que l'extREM ${ }^{\circledR}$ seront caractérisés par le Centre technique d'homologation de l'instrumentation en radioprotection (CTHIR) de l'IRSN en2002, 2006 et 2007. Le Cogebadge ${ }^{\circledR}$ reste en 2010 le dosimètre poitrine de référence pour le laboratoire.

\subsubsection{4. ... et les machines d'exploitation}

Parallèlement à l'évolution des dosimètres, le laboratoire participera à la mise au point des différentes machines d'exploitation: celles destinées à charger et décharger les films et cartes dans le badge et celles qui conditionnent sous enveloppe vinyle les badges poitrine et poignet. Il y aura plusieurs génération de machines, 
toutes resteront des prototypes, pièces uniques qu'il faudra mettre au point à chaque évolution, sans pour autant que le laboratoire ne s'arrête d'exploiter... Afin de respecter les délais règlementaires pour produire des résultats de dosimétrie, certaines journées devant les machines se sont prolongées pour certains d'entres nous...

Les machines de chargement/déchargement du badge assemblent les objets et au passage du badge, de la carte (et du film), lisent les numéros identifiant chaque objet afin d'attribuer de façon univoque le dosimètre à un porteur.

Pour les évolutions de la « mécanique » des dosimètres, la fabrication du badge, des différentes machines, le laboratoire s'appuiera sur les compétences de la même société.

Dès 1987, les grandes orientations du laboratoire sont décidées. L'organisation est en place, une équipe réalise la partie exploitation des dosimètres individuels, l'autre équipe a en charge la métrologie, les évolutions techniques et la lecture des dosimètres. Les moyens techniques sont définis. La dosimétrie par thermoluminescence est en place, pour quelques temps encore jointe au film, le Cogebadge $^{\circledR}$ a remplacé le boitier DM6 et le dosimètre poignet cherche sa technologie optimale jusqu'à s'arrêter sur l'extREM ${ }^{\circledR}$.

Le principe des machines d'exploitation des dosimètres (chargement, déchargement de films et cartes TLD, conditionnement sous enveloppe PVC des badges poitrine et poignet) est figé, des avancées technologiques jusqu'en 2000 façonneront « le design » actuel des machines. La production de dosimètres croît régulièrement avec un maximum en 1994, décroît jusqu'en 2003 et se stabilise jusqu'en 2006 (figure 11.15).

\subsubsection{Les accréditations et les évolutions règlementaires}

Alors que les fondements techniques et technologiques sont en place, le laboratoire se lance dans un nouveau challenge cette fois davantage tourné vers le management de la qualité. En 1998, le laboratoire décide de formaliser son savoir-faire et de valoriser ses outils de métrologie des rayonnements. Le laboratoire se lance dans une démarche d'accréditation COFRAC pour ses prestations d'étalonnage des « rayonnements ionisants »: rédaction de procédures, de modes opératoires et autres notes techniques. Il faut coucher sur le papier tout ce que l'on fait et se tenir à faire ce que l'on écrit. Tout ce qui concerne les moyens de métrologie doit être tracé, raccordé au système international, suivi dans le temps. Deux ans seront nécessaires à l'équipe du laboratoire pour peaufiner le système tout en continuant la dosimétrie individuelle. En mai 2000, c'est l'obtention de l'accréditation COFRAC pour réaliser des étalonnages en photons et neutrons. 
POPULATION ANNUELLE SURVEILLEE

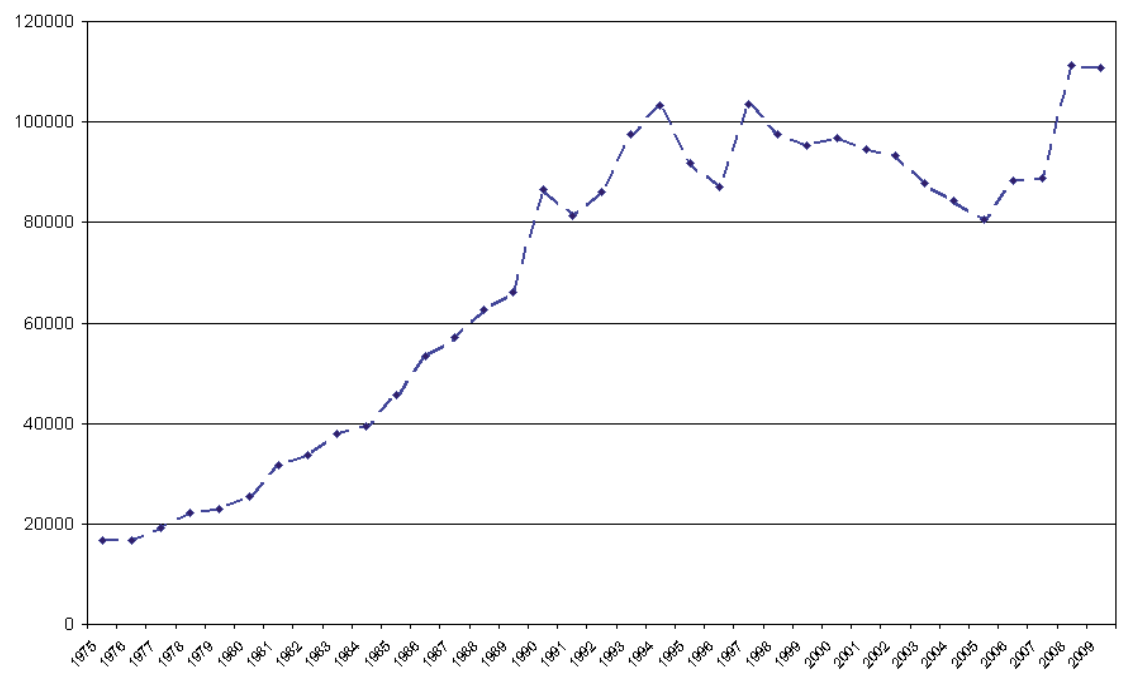

Figure 11.15 - Évolution du nombre de dosimètres de 1976 à 2009.

Il faut dès lors maintenir le système. Même si le rythme est pris, ce sont des audits internes ou COFRAC qui sont réalisés tous les ans et cela n'est pas neutre dans l'activité du laboratoire. D' autant qu'en 2003, c'est la règlementation qui nous conviera à une nouvelle accréditation...

Jusqu'à la parution du décret 2003-296 du 31 mars 2003, le laboratoire de dosimétrie de La Hague, comme les autres laboratoires français (exception faite du laboratoire de l'OPRI, aujourd'hui IRSN), fonctionnait au travers d'une «autorisation» formalisée par un arrêté délivré par le ministère du Travail, de l'emploi et de la formation professionnelle, les décrets n ${ }^{\circ} 75-306$ du 28 avril 1975 et $n^{\circ} 86-1103$ du 2 octobre 1986 étaient alors en vigueur. Cette autorisation est délivrée pour cinq ans. Elle limite le laboratoire au seul suivi du personnel travaillant sur le site. Pour obtenir cette autorisation, le laboratoire doit obtenir des résultats de dosimétrie compatibles lors de l'intercomparaison organisée par l'OPRI. Le décret 2003-296 du 31 mars 2003 exigera pour chaque laboratoire un agrément afin de pouvoir assurer la surveillance individuelle de l'exposition externe des travailleurs. Les modalités de cet agrément sont définies dans l'arrêté du 6 décembre 2003 aujourd'hui modifié par l'arrêté du 21 décembre 2007. L'ASN délivre cet agrément. Pour être agréé, un laboratoire doit être accrédité. Il doit obtenir un avis 
H. TRUFFERT

favorable de l'IRSN quant à l'adéquation des matériels et des méthodes utilisés et avoir des résultats compatibles aux intercomparaisons organisées par l'IRSN. L'organisation du laboratoire doit être telle que son indépendance soit garantie. Le dosimètre utilisé doit être « caractérisé ».

Le laboratoire obtiendra son accréditation COFRAC en avril 2004 conformément à la norme ISO/CEI 17025 et le document COFRAC LAB REF 13. La caractérisation du Cogebadge ${ }^{\circledR}$ avait été faite en 2002. Elle sera refaite en 2006 sans le film avant que celui-ci soit retiré et en 2007 pour la partie neutronique. Cette même année, le dosimètre poignet sera caractérisé également. Les autres éléments (l'adéquation des matériels et des méthodes, l'indépendance et les résultats de l'intercomparaison), étant jugés conformes par l'IRSN et les évaluateurs du COFRAC, le laboratoire obtiendra son agrément à la fin de l'année 2004, agrément qui sera renouvelé en décembre 2007. Entre temps, la publication de l'arrêté du 30 décembre 2004 créera l'événement en fixant des seuils d'enregistrement inférieurs à ceux atteignables par le film : « la plus petite dose non nulle enregistrée ne peut être supérieure à $0,10 \mathrm{mSv}$ ». Le dosimètre photographique avait vécu... Dès lors, les doses calculées avec les dosimètres thermoluminescents sont règlementaires et sont la base du suivi dosimétrique du salarié. Le laboratoire retirera définitivement le film du Cogebadge ${ }^{\circledR}$ en octobre 2006.

Le Laboratoire agréé se doit également de communiquer tous les mois les résultats de dosimétrie passive concernant les salariés qu'il suit à SISERI.

Le système SISERI a été mis en place par l'IRSN (voir chapitre 13). Son objectif est de constituer une base nationale qui centralise et conserve l'ensemble des résultats des mesures individuelles de l'exposition des travailleurs. Ces données sont accessibles par Internet aux médecins du travail et personnes compétentes en radioprotection, dans un but d'optimisation de la surveillance médicale et la radioprotection des travailleurs.

Voici en résumé le processus du Laboratoire en place aujourd'hui pour réaliser la dosimétrie externe règlementaire des travailleurs (figure 11.16).

\subsection{Conclusion}

Après des années à forts enjeux techniques pour bâtir la structure du laboratoire afin de réaliser la dosimétrie, il faut désormais associer un management de la qualité et une gestion des compétences des personnels pour maintenir l'agrément ( $c f$. la norme ISO 17025). Cet agrément a quelque peu changé la vie du Laboratoire. Il a permis entre autre au laboratoire d'élargir le champ de ses prestations dosimétriques à des personnes provenant d'entités autres que celles du site de la Hague et ce depuis 


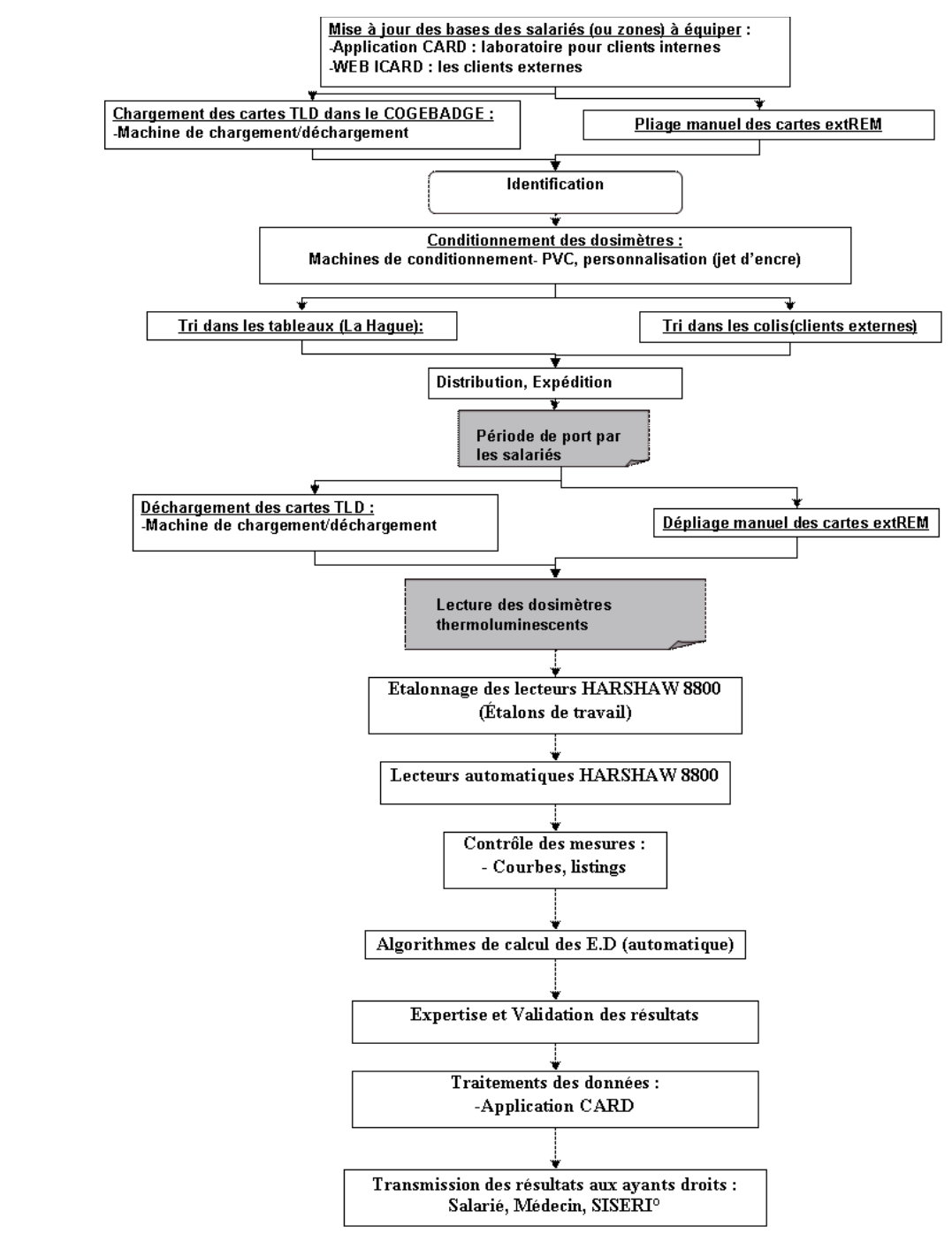

Figure 11.16 - Organigramme décrivant le processus du laboratoire.

2006. Mais le Laboratoire reste un « mini » laboratoire de dosimétrie au regard du nombre de dosimètres traités par rapport à d'autres. La capacité actuelle du Laboratoire permet de traiter 120000 Cogebadge ${ }^{\circledR}$ par an (dosimètres du personnel, de zone..) et 40000 extREM $^{\circledR}$. Cette nouvelle activité est aussi synonyme de nouvelles tâches comme par exemple l'expédition des dosimètres, la relation avec 
les différents clients... et de nouveaux développements telle qu' une interface Web afin que les clients externes puissent faire les commandes de dosimètres ou les modifications concernant leurs personnels à équiper. Cette fois, il a fallu revoir l'organisation du Laboratoire pour tenir compte de ces missions que la proximité des salariés du site nous avait fait ignorer jusqu'alors.

\section{Remerciements}

Je ne peux pas écrire ces quelques lignes sur la dosimétrie de La Hague sans rendre hommage à celui qui a œuvré pour que le laboratoire soit ce qu'il est aujourd'hui, René Prevot. Il fut responsable du laboratoire de 1972 à 1993, date à laquelle il me laissera sa place. C'est lui qui m'a appris « la dosimétrie » et c'est avec lui que j'ai participé aux différents développements et évolutions que le laboratoire a réalisés. 